\title{
The Value of CA19-9 After Irreversible Electroporation for Pancreatic Cancer
}

\author{
CHRISTOPHER MÅNSSON, ANDERS NILSSON, JOZEF URDZIK and BRITT-MARIE KARLSON \\ Department of Surgical Sciences, Uppsala University, Uppsala, Sweden
}

\begin{abstract}
Background/Aim: Carbohydrate antigen 19-9 (CA19-9) is a tumor marker for pancreatic cancer. Irreversible electroporation (IRE) is an experimental treatment modality for pancreatic cancer. The aim of this study was to evaluate whether percutaneous IRE lowers the CA19-9 level in pancreatic cancer and whether this correlates with improved overall survival. Patients and Methods: Seventy-one patients with locally advanced pancreatic cancer or local recurrence after resection were treated. Patients with missing data, metastatic disease and normal serum CA19-9 before IRE were excluded. This left 35 cases for analysis. Results: The median CA19-9 did not decrease in the cohort after IRE treatment (282 U/ml before versus $315 \mathrm{U} / \mathrm{ml}$ after; $p=0.80$ ). The 25th percentile of patients with the best CA19-9 response had improved overall survival compared to the 25th percentile with the worst response (mean 13.1 versus 8.1 months, respectively; $p=0.01)$. Conclusion: IRE did not lower the level of CA19-9 in pancreatic cancer cases. However, a response in CA19-9 was correlated with improved survival.
\end{abstract}

Pancreatic cancer is a severe disease with poor prognosis even with optimal treatment. It is the fourth leading cause of death from cancer although its incidence is only in the 10th place (1). Several experimental treatment modalities have been tested to try to improve prognosis. Irreversible electroporation (IRE) is one of these and it has shown some promise regarding prognosis for locally advanced pancreatic cancer (2).

Carbohydrate antigen 19-9 (CA19-9) is a molecule that is synthesized in humans by some epithelial cells, among them pancreatic and bile duct cells. It is found elevated in some

This article is freely accessible online.

Correspondence to: Christopher Månsson, Department of Surgical Sciences, Uppsala University, 75185 Uppsala, Sweden. Tel: +46 186114505. Fax: +46 186114508, e-mail: christopher. mansson@surgsci.uu.se

Key Words: Pancreatic neoplasms, electroporation, interventional ultrasonography, CA19-9. cancer types, including pancreatic cancer, and is therefore used as a tumor marker for pancreatic cancer in several settings (3). Postoperative normalization of serum CA19-9 after pancreatic resection for pancreatic cancer correlates with an improved prognosis compared to an increase or lower but still elevated value (4). CA19-9 can help predict which patients are suitable for resection after induction therapy for locally advanced pancreatic cancer (5). Pre- and post-treatment CA19-9 can also be used to evaluate the effect of chemotherapy on pancreatic cancer (6).

A study by Lin et al. showed the CA19-9 value after IRE treatment of pancreatic cancer to be lower than before treatment (7). We used IRE as an experimental treatment of pancreatic cancer since 2011 and have published some of our results (8-10). In this study, we analyzed the effect on CA199 after IRE in our experience.

The primary aim was to evaluate the response in CA19-9 level after IRE treatment, and the secondary aim was to estimate the impact of CA19-9 response on overall survival.

\section{Patients and Methods}

Patients with locally advanced pancreatic cancer, before and after chemotherapy, or with local recurrence after pancreatic resection, were treated. An IRE machine called Nanoknife (AngioDynamics Inc., Queensbury, NY, USA) was used. All patients had histologically proven pancreatic adenocarcinoma and had been discussed at multidisciplinary team conference prior to inclusion. All patients that were referred to our center that met the inclusion criteria were invited to participate. Exclusion criteria was resectable tumor, metastases, implanted electronic devices, ASA-score IV, expected survival $<3$ months, pregnancy, epilepsy, severe heart disease, and tumor diameter $>5.0 \mathrm{~cm}$, for the patients' basic characteristics please see Table I. All treatments were performed percutaneously by ultrasound guiding by the same interventional radiologist. The treatments were performed under general anesthesia with deep neuromuscular block (post-tetanic count of 0 ). One month after IRE treatment, an ultrasound examination was performed to look for complications and metastases. Pre- and post-treatment CA19-9 levels were measured. The 1-month time point was selected for determining the postoperative CA19-9 level since no chemotherapy was given between the IRE treatment and ultrasound examination at 1 month. Thus, the IRE treatment was the only thing that could have affected the cancer and the CA19-9 level. Since not 
all pancreatic cancers express CA19-9, those patients that had a normal CA19-9 level before IRE treatment were not included.

The study was approved by the Uppsala Regional Ethics Committee, Uppsala, Sweden (Dnr 2013/254).

\section{Results}

During the study period, 71 patients were treated with IRE. Due to missing CA19-9 values for two patients before treatment and 14 patients after treatment, these patients were excluded. The most common reasons for the missing CA19-9 values were early recurrent disease or complications 1 month after IRE treatment. Ten patients had metastases at the 1month ultrasound and were also excluded from analysis since they most likely had metastases that were undetected prior to the treatment. Thus, in their cases, local IRE treatment was not a factor and their elevated pre-operative level of CA19-9 might have been from these metastases. Ten patients who did not have a pathological CA19-9 level before IRE treatment were also excluded. Data for 35 patients therefore remained for analysis. For a summary of the patient selection procedure, please see Figure 1.

CA19-9 level. There was no decrease in CA19-9 at 1 month after IRE treatment. The median CA19-9 level before IRE treatment was $282 \mathrm{U} / \mathrm{ml}$ and after the treatment was slightly higher at $315 \mathrm{U} / \mathrm{ml}(p=0.80$; Figure 2$)$. However, there was a large difference between the patients' response in CA19-9.

Changes in CA19-9. The effect of changes in CA19-9 level after IRE treatment on survival was evaluated. Since a small change in CA19-9 has no clinical implications and the threshold for any effects are unknown, we considered the 25th percentiles with the best and the worst CA19-9 response. This meant a decrease or increase of approximately $50 \%$ of the initial CA19-9 value. We found that those with the best response in CA19-9 had an improved mean overall survival of 13.1 versus 8.1 months for those with the worst response (log-rank $p=0.01$; Figure 3 ).

\section{Discussion}

We cannot confirm the hypothesis that IRE treatment lowers the median CA19-9 as was previously postulated by Lin et al. (7). In fact, in our study, the median CA19-9 level was slightly higher after treatment. The reason for this finding is not clear. It might be that IRE treatment does not affect the tumor in such a way that the CA19-9 value decreases. It may also be that many of the patients, even though they had no radiological signs of metastatic disease, had micrometastases, which are not affected by the IRE treatment. It may also, in fact, be that in some cases, the CA19-9 level is elevated by IRE treatment.
Table I. Basic patient characteristics before irreversible electroporation treatment.

\begin{tabular}{lc}
\hline & Value \\
\hline Gender, n (\%) & \\
$\quad$ Male & $17(49)$ \\
Female & $18(51)$ \\
Age, years & $66(61-74)$ \\
$\quad$ Median (IQR) & $31(89)$ \\
Type of tumor, n (\%) & $4(11)$ \\
LA & \\
Local recurrence & $23(66)$ \\
Location of tumor, n (\%) & $12(34)$ \\
Caput & \\
Corpus/cauda & $35(27-40)$ \\
Max tumor diameter, mm & \\
Median (IQR) &
\end{tabular}

IQR, Interquartile range; LA, locally advanced.

Patients with a decrease in CA19-9 level after IRE treatment had a better prognosis. This implies the use of CA19-9 level before and 1 month after IRE treatment as a prognostic marker. This might indicate which patients are suitable for an aggressive approach with chemotherapy post IRE with the intent of reaching a setting for radical resection. To our knowledge, this is the first study that has investigated CA19-9 as a prognostic marker after IRE treatment. The fact that all patients were treated at a single center by the same interventional radiologist can be seen as positive. The fact that between the two CA19-9 measurements no chemotherapy was given which could have influenced the CA19-9 level can also be seen as positive.

One weakness of the study was not utilizing diagnostic laparoscopy before the IRE treatment, as Martin et al. did (2). Had we used laparoscopy, the results might have been different, since we would have been better able to rule out metastatic disease. Moreover, unlike Martin et al. (2), we performed all procedures percutaneously, not with open surgery. This avoids complications related to open surgery but the open operation is another tool with which to rule out metastases before IRE treatment.

\section{Conclusion}

IRE did not lower the CA19-9 level in patients with pancreatic cancer. However, a response in CA19-9 was correlated with improved survival.

\section{Conflicts of Interest}

Anders Nilsson has received travel funding for lectures from Angiodynamics, the company that produces the IRE machine. 


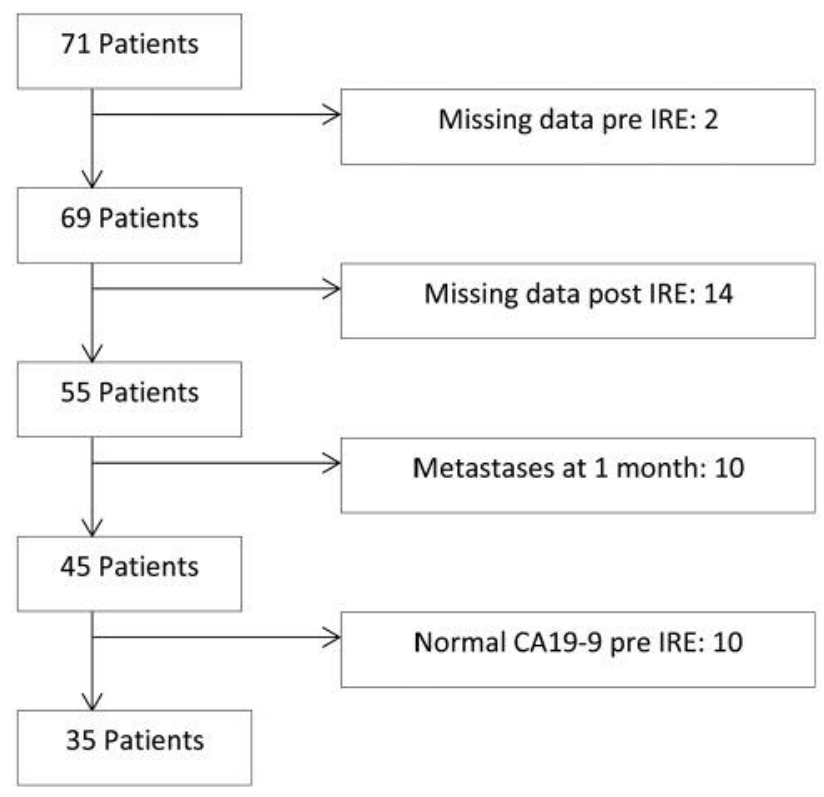

Figure 1. CONSORT diagram showing which patients were included in this study. IRE, Irreversible electroporation.

\section{Authors' Contributions}

CM: Study design, data collection, statistics, preparation of article, final approval of article. AN: Study design, data collection, preparation of article, final approval of article. JU: Study design, statistics, preparation of article, final approval of article. BMK: Study design, data collection, preparation of article, final approval of article

\section{Acknowledgements}

The Authors thank Mandy Trickett for language editing.

\section{References}

1 Brunner TB and Seufferlein T: Pancreatic cancer chemoradiotherapy. Best Pract Res Clin Gastroenterol 30(4): 617-628, 2016. PMID: 27644909. DOI: 10.1016/j.bpg.2016.08.001

2 Martin RC, 2nd, Kwon D, Chalikonda S, Sellers M, Kotz E, Scoggins C, McMasters KM and Watkins K: Treatment of 200 locally advanced (stage III) pancreatic adenocarcinoma patients with irreversible electroporation: Safety and efficacy. Ann Surg 262(3): 486-494, 2015. PMID: 26258317. DOI: 10.1097/ sla.0000000000001441

3 Scara S, Bottoni P and Scatena R: CA19-9: Biochemical and clinical aspects. Adv Exp Med Biol 867: 247-260, 2015. PMID: 26530370. DOI: 10.1007/978-94-017-7215-0_15

4 Xu HX, Liu L, Xiang JF, Wang WQ, Qi ZH, Wu CT, Liu C, Long J, Xu J, Ni QX and Yu XJ: Postoperative serum CEA and CA125 levels are supplementary to perioperative CA19-9 levels in predicting operative outcomes of pancreatic ductal

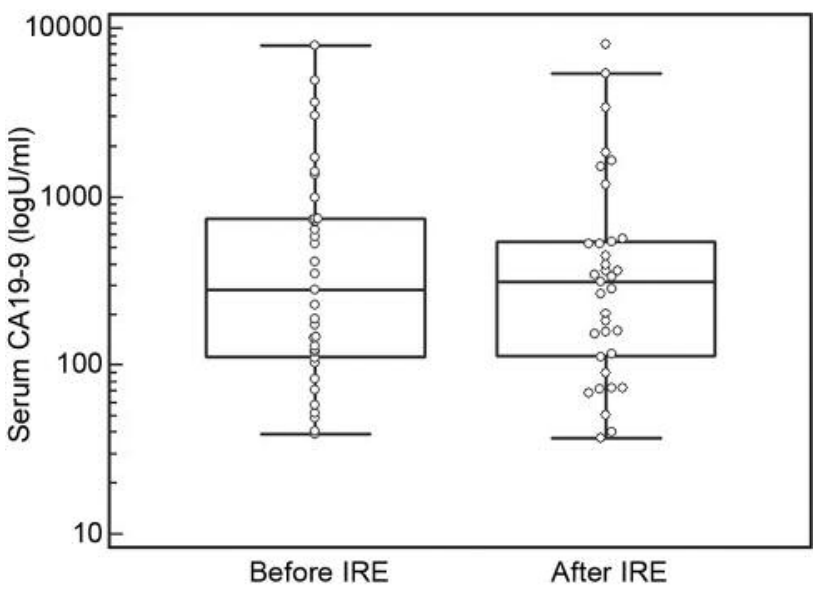

Figure 2. Boxplot of CA19-9 levels before and 1 month after irreversible electroporation (IRE). The Y-axis represents logarithmic serum CA19$9(U / m l)$.

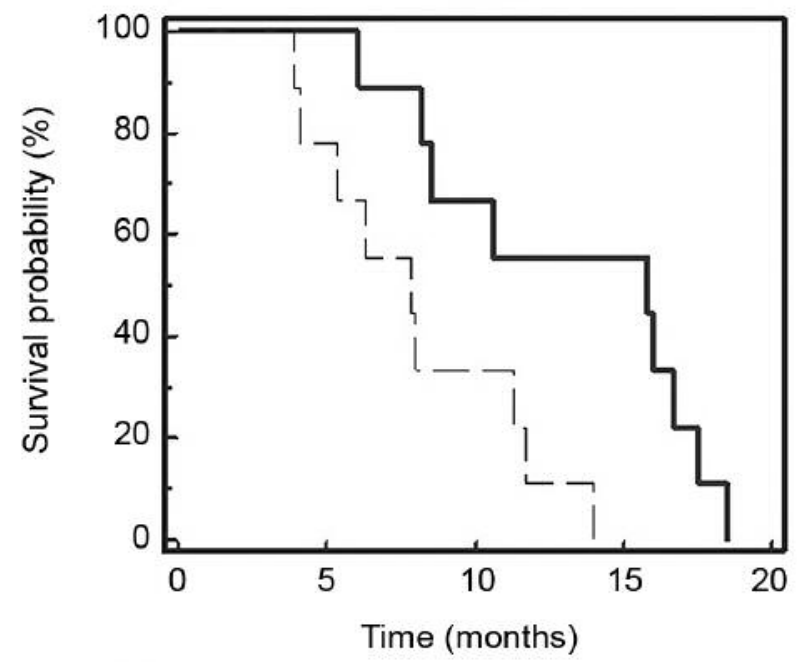

\section{Response

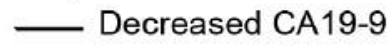 \\ - - Increased CA19-9}

Figure 3. Kaplan-Meier curve showing survival for the 25th percentile of patients with best (decrease, $N=9$ ) and worst (increase, $N=9$ ) responses in CA19-9 level at 1 month after irreversible electroporation (IRE).

adenocarcinoma. Surgery 161(2): 373-384, 2017. PMID: 27838 102. DOI: $10.1016 /$ j.surg. 2016.08 .005

5 van Veldhuisen E, Vogel JA, Klompmaker S, Busch OR, van Laarhoven HWM, van Lienden KP, Wilmink JW, Marsman HA and Besselink MG: Added value of CA19-9 response in predicting resectability of locally advanced pancreatic cancer following induction chemotherapy. HPB 20(6): 605-611, 2018. PMID: 29475787. DOI: 10.1016/j.hpb.2018.01.001 
6 Colloca G, Venturino A and Guarneri D: CA19-9-related tumor kinetics after first-line chemotherapy of patients with advanced pancreatic cancer: A monoinstitutional experience. Med Oncol 33(9): 103, 2016. PMID: 27522503. DOI: 10.1007/s12032-0160817-x

7 Lin M, Liang S, Wang X, Liang Y, Zhang M, Chen J, Niu L and $\mathrm{Xu}$ K: Percutaneous irreversible electroporation combined with allogeneic natural killer cell immunotherapy for patients with unresectable (stage III/IV) pancreatic cancer: A promising treatment. J Cancer Res Clin Oncol 143(12): 2607-2618, 2017. PMID: 28871458. DOI: 10.1007/s00432-017-2513-4

8 Mansson C, Brahmstaedt R, Nilsson A, Nygren P and Karlson BM: Percutaneous irreversible electroporation for treatment of locally advanced pancreatic cancer following chemotherapy or radiochemotherapy. Eur J Surg Oncol 42(9): 1401-1406, 2016. PMID: 26906114. DOI: 10.1016/j.ejso.2016.01.024
9 Mansson C, Bergenfeldt M, Brahmstaedt R, Karlson BM, Nygren $\mathrm{P}$ and Nilsson A: Safety and preliminary efficacy of ultrasound-guided percutaneous irreversible electroporation for treatment of localized pancreatic cancer. Anticancer Res 34(1): 289-293, 2014. PMID: 24403476.

10 Mansson C, Brahmstaedt R, Nygren P, Nilsson A, Urdzik J and Karlson BM: Percutaneous irreversible electroporation as firstline treatment of locally advanced pancreatic cancer. Anticancer Res 39(5): 2509-2512, 2019. PMID: 31092446. DOI: 10.21873/ anticanres.13371 